\title{
Clinical outcomes of Percutaneous Endoscopic Debridement and Drainage combined with Percutaneous Pedicle Screw Fixation for the treatment of Infective Spondylodiscitis
}

Jingsong Liu

First Affiliated Hospital of Harbin Medical University

Daqian Liu

First Affiliated Hospital of Harbin Medical University

\section{Gang An}

First Affiliated Hospital of Harbin Medical University

\section{Ying Guan}

First Affiliated Hospital of Harbin Medical University

\section{Xu Shi}

First Affiliated Hospital of Harbin Medical University

\section{Ran Wan}

First Affiliated Hospital of Harbin Medical University

\section{Yiming Fan}

First Affiliated Hospital of Harbin Medical University

\section{YanSong Wang ( $\sim 1538609332 @ q q . c o m)$}

First Affiliated Hospital of Harbin Medical University

\section{Research article}

Keywords: infectious spondylitis; percutaneous endoscope debridement; percutaneous drainage; postoperative irrigation and drainage邓percutaneous pedicle screw; sensitive antibiotics

Posted Date: May 4th, 2020

DOl: https://doi.org/10.21203/rs.3.rs-23766/v1

License: (1) This work is licensed under a Creative Commons Attribution 4.0 International License. Read Full License 


\section{Abstract}

Background: The morbidity of infectious spondylitis is increasing year by year in the elderly. Due to difficulties in coping with open surgery, we aim at presenting a treatment method for infectious spinal inflammation by the combination of Percutaneous Endoscopic Discectomy and Drainage (PEDD) and Percutaneous Pedicle Screw Fixation (PPSF) which obtained satisfactory outcomes in this study.

Method: We have retrospectively analyzed 33 patients who had suffered from lumbar infectious spondylitis and undergone PEDD combined with the PPSF treatment. Biopsy specimen was taken from each of them during their operation and the specimen was submitted without delay for microculture. The dynamic change of patient clinical symptoms and their satisfaction degree were assessed by careful physical examination, regular serologic test, imaging study, Odom's criteria, Frankel score, and visual analog scale (VAS).

Result: Among the 33 patients, 22 (66.7\%) had excellent symptom relief and the remaining 11 (33.3\%) had good symptom relief after PEDD treatment according to Odom's criteria. Additionally, PPSF was performed on 5 patients in the second stage, and yielded satisfactory result. There were no severe surgery-related complications. The microculture from 24 patients $₫ 72.7 \%$ obtained from the operation were positive.

Conclusion: PEDD and PPSF were under the influence of local anesthesia, which had lower requirements of patients' general condition comparing to the traditional open surgery. In addition, PEDD obtained higher positive rate of microorganisms cultured comparing to other minimally invasive methods. PPSF was performed at the second stage to alleviate pain and further improve vertebral stability.

\section{Background}

Infectious spondylitis is a rare and severe spinal disease that can be classified as suppurative, nonspecific, or atypical (mycobacterium tuberculosis or Brucella infection). According to relevant literature, the incidence of acute hematopoietic non-tuberculous spinal osteomyelitis is 5-5.3 per million patients per year, affecting mostly male. A French study showed that the annual incidence of spinal disc inflammation was 2.4 per 100,000 years, with the number of patients over the age of 70 nearly tripled[1]. In recent years, owing to the development of computed tomography (CT), magnetic resonance imaging (MRI) and other imaging technologies, the diagnostic rate of diseases has improved. The increase in incidence of infectious spondylitis is often associated with older age, intravenous drug abuse, poor immune status, or postoperative infection. Older adults are more likely to develop spondylitis due to poor physical conditions and immune status. The use of implantable devices, such as central venous catheters, also increase the risk of getting blood-borne infections[2-6]. Lumbar spine is the primary region affected by infectious spondylitis, followed by thoracic, cervical, and sacral levels[7]. Typical clinical symptoms include severe low back pain with or without sciatica, which has no obvious early symptoms. 
Clinical diagnosis is confirmed by combining CT, MRI and other imaging examinations, erythrocyte sedimentation rate (ESR), $C$ reactive protein (CRP) and other laboratory examination results. Infectious spondylitis is essentially a medical disease and is often treated conservatively. The primary procedure of standard treatment for infectious spondylitis is locating the infected region, culturing the infected tissue, and applying effective antibiotic therapy based on the culturing results, with proper resting and external fixation. Surgical treatment was performed only for patients with unsatisfactory conservative treatment, failed infection control, progressive neurological impairment, and progressive spinal deformity. Common surgical procedures include anterior decompression and anterior decompression combined with posterior fixation and fusion[8-11]. Because the operation needs to be performed under general anesthesia, which poses potential threat to elderly patients with other medical diseases or are in poor physical conditions, minimally invasive techniques such as ultrasound or CT-guided puncture and drainage were given the priority over surgery. With the rapid development of minimally invasive intervertebral foramen technique in spine surgery in recent years, percutaneous foramen discectomy with lavage and drainage has become a new minimally invasive treatment method for infectious spinal diseases. However, the minimally invasive techniques mentioned above have not been applied to the stabilize the spine. Long-term vertebral instability and deformity may easily result in further pain or nerve compression symptoms due to vertebral destruction. In this study, patients received local anesthesia through lenticels microscopically and intervertebral disc lesion clearance lavage Drainage PEDD combined with PPSF to treat infectious spondylitis, and 2011.09 -2018.09 in our hospital during the infectious spondylitis treated with surgery were retrospectively analyzed.

\section{Methods}

\section{General Information}

33 patients ( 20 males and 13 females, aged $29-89$ years, with an average age of $71.03 \pm 13.91$ years) admitted to our department from September 2011 to September 2018 were selected. All patients were assessed by detailed medical history, physical examination and imaging examination. Inclusion criteria for patients: 1) low back pain with or without sciatica; 2) increased ESR and CRP; 3) X-ray, CT and MRI showed obvious narrowing of intervertebral space, or vertebral endplate destruction with or without intervertebral instability. 4) single segment infection with or without paravertebral abscess and epidural abscess. Exclusion criteria: 1) postoperative spinal infection; 2) other spinal history or surgery history; 3 ) multi-segmental infection; 4) other spinal disorders. All 33 patients exhibited limited mobility and bed rest caused by low back pain. Twelve patients experienced pedal, reduced leg strength, and sciatica. Only 10 patients showed positive for preoperative blood culture or needle aspiration culture, and serological examination showed that CRP and ESR of all 33 patients had significantly increased, which confirmed the diagnosis of infectious spondylitis supplemented by $\mathrm{CT}, \mathrm{MRI}$ and other imaging results. According to the American Society of Anesthesiologists (ASA), 11 patients were classified as level II, 20 as level III, and the remaining 2 patients were in good health. Most patients stayed in bed with a brace support while receiving antibiotic treatment for at least three weeks. 10 patients were given sensitive antibiotics based on culturing results. Patients with suspected tuberculosis were treated with anti-tuberculosis drugs. The 
rest of the patients were prescribed drugs based on our experience. For patients whose infection showed no sign of being controlled according to the periodic re-examination with CRP, ESR, CT, MRI and other diagnostic imaging techniques during the treatment period, they were considered for surgical treatment. Among them, infections were detected at L2/3 in 8 cases, L3/4 in 12 cases, L4/5 in 10 cases, and L5/S1 in 3 cases. Imaging examination of 16 patients suggested paravertebral abscess or epidural abscess. (table 1).

\section{Surgical Techniques}

\section{PEDD}

The patient was in a prone position on a radiolucent frame suitable for fluoroscopy. After routine disinfection, the responsible segments were defined under the guidance of intraoperative X-ray. Local anesthesia with $0.5 \%$ lidocaine was performed at an area $8-12 \mathrm{~cm}$ away from the median line of the responsible gap level, and the puncture needle was obtruded down at an angle of 15-30 degrees from the sagittal plane, and the infiltration anesthesia was conducted layer by layer. The needle tip was inserted to the inner edge of the pedicle and the intervertebral space, as guided by the anteroposterior and lateral images of the intraoperative X-ray. The guide wire was inserted through the needle, which was subsequently pulled out, followed by incision of about $1 \mathrm{~cm}$ of the skin at the puncture point. A dilator and a cannulated sleeve were delivered through the wire and sent sequentially into the disc center. Intraoperative X-ray confirmed the correct placement of the channel. Then the dilator was removed, and the endoscope was introduced. With the aid of the scope, the infected intervertebral disc tissue was located and extracted by nucleus forceps. Forceps, bone-biting forceps and bipolar electrocoagulation were used to remove as much tissues as possible. After adjustment of the position and angle of the channel, the endoscope was removed, and the adjacent vertebral endplate was scraped off as much as possible using the scraping spoon. 3.5\% povidone iodine solution was prepared using standard saline, and at least $10000 \mathrm{ml}$ of such solution was used for lavage. The endoscope was re-inserted to check the completeness of the debridement. A disposable epidural anesthesia catheter and a drainage tube with a diameter of about $3.5 \mathrm{~mm}$ were inserted. The wound was sutured and normal saline was injected through the anesthesia catheter. Upon confirmation of smooth drainage, the two tubes were fixed on the skin. Phase I PPSF or phase II PPSF was selected based on patient's choice and physical condition.

After the operation, saline was used and corresponding sensitive antibiotics were applied, based on the culture result, for continuous irrigation for 2-12 weeks. After the rinsing solution was clear, the drug delivery tube was removed and a single cavity tube was used for drainage. The drainage tube was removed after the daily drainage volume fell below $10 \mathrm{ml}$ for 3 consecutive days. Patients were allowed to move postoperatively.

\section{PPSF}

Following the same procedure as PEDD, the patient was in a prone position on a radiolucent frame suitable for fluoroscopy. After routine disinfection, the responsible segment was identified using 
intraoperative X-ray, and the insertion point was identified using the anteroposterior X-ray. Four paravertebral skin incisions were made, each of them was about $1.5 \mathrm{~cm}$ long. Then, the insertion point under the c-arm was reconfirmed, and pedicle screw was fixed into the pedicle according to the image displayed by the c-arm. Two titanium alloy bars of appropriate length were pre-bent and inserted into the openings of the bilateral screws. The locking nail rod system was used, and confirmation of insertion position was performed using X-ray. This was followed by repeated irrigation and layer by layer occluding.

\section{Evaluation Criteria}

Preoperative physical examination was performed to learn about patient's medical history. Charlson Comorbidity Index was used to assess the patient's physical conditions. Changes in preoperative and postoperative pain were assessed by Visual Analogue Scale (VAS). Changes in neurological function were assessed by Frankel grading. Cobb Angle was measured to evaluate kyphosis progression. Postoperative Odom's criteria were used to assess patient's satisfaction. Laboratory examinations were performed one day, one week, one month and three months postoperatively. Although ESR and CRP were non-specific indicators, both were elevated in almost all patients with infectious spinal inflammation, and their changes corresponded with changes in the course of the disease. Microbiological culture was performed for all pathologies for samples collected from the operation.

\section{Statistical Analysis}

SPSS 17.0 software (chicago.il, American) was used for input of CRP, ESR, and VAS scores, plus other data before and after surgery. Paired $t$ test was performed to analyze the differences and correlations between preoperative and postoperative parameters, with $p<0.05$ considered to be statistically significant.

\section{Results}

The average operation time of PEDD was $66.5 \pm 13.5 \mathrm{~min}$, and the blood loss was $5-20 \mathrm{ml}$. The mean operation time of PPSF was $79 \pm 9.7 \mathrm{~min}$ and the blood loss was $10-25 \mathrm{ml}$. Among the 33 patients, 5 patients received PPSF three days after PEDD according to their will or due to difficulty in tolerating the long-time operation. The remaining 28 patients received PPSF immediately after PEDD. According to the Odom's criteria, all 28 patients who underwent the combined operation in the first stage were satisfactory with the surgical effect, among which 22 were excellent and 6 were good. After receiving PEDD, 3 of the remaining 5 showed fair and 2 showed good. 3 days after PPSF, all of them obtained good results (Table 2). VAS were recorded for patients one day, one week, one month and three months after the operation, with all the patients showing significant decrease in the score $(p<0.001)$. It's worth noting that the VAS was further decreased in 5 patients after the second stage PPSF (Fig. 1). CRP and ESR values decreased gradually $(p<0.001)$, and returned to normal levels on average in January (Figs. 2 and 3 ). All 12 patients with neurological symptoms showed significant improvement in Frankel classification 3 months after the surgery (Table 3). Of the 33 patients, 24 obtained positive culture results for 
intraoperative pathology (Table 4 and Table 5) and were replaced with drug-sensitive antibiotics accordingly, while the rest of the patients with negative culture continued to receive empirical antibiotics. These 33 patients were followed up for an average of $7.84 \pm 4.29$ months ( $3-24$ months) after the surgery, and X-ray imaging showed an average increase of $3.9 \pm 6.7^{\circ}$ in the local Cobb Angle, and $90.9 \%$ of the patients had vertebral bridge or fusion. No recurrence was observed during follow-up, and no secondary anterior or posterior open surgery was performed. No surgical complications occurred in any of the patients.

\section{Discussion}

Currently, there have been different opinions concerning whether conservative or surgical treatment should be applied for infectious spondylitis. Surgery was performed only in patients with unsatisfactory conservative treatment, failed infection control, progressive neurological impairment, and progressive spinal deformity[8-11]. In this study, all patients underwent at least 3 weeks of conservative treatment, and still experiencing persisted symptoms. The number of infectious spondylitis patients is increasing year by year, and due to medical development and population aging, the infected population gradually shifts from teenagers and middle-aged people to the elderly. Almost half of the infected people were aged over 50[12]. The main surgical methods for infectious spinal inflammation are anterior decompression and anterior decompression combined with posterior fixation and fusion, both of which require general anesthesia and are accompanied by large blood loss and long operation time. Pee performed anterior lesion removal and bone graft fusion and posterior pedicle screw internal fixation in 60 patients, with an average operation time of $263.8 \pm 83.0 \mathrm{~min}$ and an average blood loss of $810 \pm 49.6 \mathrm{ml}$ [13]. For elderly infected with spinal inflammation, due to their poor physical condition and autoimmune state, it is difficult for them to tolerate the trauma caused by traditional surgery, large blood loss and long anesthesia time. It has been reported that anterior fusion surgery has a $15 \%$ incidence of perioperative complications[14] and a 4-5\% mortality[15]. In this study, the average age of the patients was $71.03 \pm$ 13.91 years old, which was generally old. Most of the patients were rated as ASA level III with one or more internal diseases including diabetes, stroke, renal failure, etc., and had been lied in bed for at least 3 weeks before surgical treatment, with poor physical condition and were unsuitable or at high risk of receiving general anesthesia. Compared with the traditional operation, PEDD and PPSF are all performed under local anesthesia, which induces a lower risk compared to general anesthesia by avoiding endotracheal intubation and thus reducing the likely incidence of postoperative pneumonia, and associated difficulty in exhaling management. Traditional open surgery requires complex and lengthy preoperative preparation and adjustment. For patients with neurological defects, PEDD can relief nerve root compression and rescue neurological function in the shortest time.

Intraoperatively, a scraping spoon was used to remove the adjacent vertebral endplate. In adults, where there is no vascular tissue in the intervertebral disc, intra-osseous infusion extends into the terminal artery at about age 30[16]. In adults, the subchondral spongiform bone is supplied with nutrients from the terminal artery, where small septic emboli may stay in and begin to proliferate, leading to infarct and subsequent osteomyelitis. As infection reaches to the subchondral space, it usually continues to spread 
to the disc, causing osteomyelitis and discitis. The infection can then spread to the adjacent vertebral endplate through the disc. Therefore, in our opinion, the removal of the endplate during the operation can make the lesion removal more thorough and contributes to a lower recurrence rate, which can't be achieved by other minimally invasive methods except for direct vision using an endoscope. At the same time, the scraping of adjacent vertebral endplates also promoted the spontaneous fusion of vertebral bodies. The primary objective of conservative treatment is to apply a large amount of antibiotics to control the infection and wait for spontaneous fusion of the adjacent vertebral bodies of the infected segment, a process that often takes 3 months[17]. However, the lesion clearance of PEDD and the vertebral stability brought by PPSF all facilitate the spontaneous fusion among vertebral bodies, enabling patients to perform early functional exercise, reduce bed immobilization, and reduce the incidence of perioperative complications such as thrombotic pulmonary embolism.

The main clinical manifestations of infectious spondylitis are intractable back pain. The primary manifestation of infectious spondylitis is the erosion of intervertebral space. Low back pain caused by infection and inflammatory stimulation can be further aggravated by slight changes in position or movement (such as turning over) due to damage to the vertebral space and endplate. Conservative treatment often relieves pain by immobilizing in bed to avoid movement between the adjacent vertebral bodies. Nasto et al. compared the differences between the treatment of infectious spondylitis with percutaneous posterior fixation and conservative treatment with traditional supports, the results showed that VAS in the fixation group were lower than those in the conservative group[12]. In our study, 33 patients had significant pain relief after surgery. It is notable that the VAS decreased from $8.20 \pm 0.83$ points to $6.20 \pm 0.44$ points for the 5 patients who received the second-stage operation after the firststage PEDD. This means after receiving PPSF in the second phase, the score further decreased to $2.40 \pm$ $0.89(p<0.005)$. We believe that PEDD can rapidly solve the patients' lower back pain by debridement and lavage of intervertebral space under the endoscope, and further clear the lesions by continuous irrigation and drainage after the catheter placement. However, other than the destruction of posterior ligament complex and paravertebral muscle caused by open surgery, all of the disc, the adjacent soft tissues and vertebral endplate were scraped in PEDD. The stability of the vertebral body was still affected before the formation of spontaneous fusion, therefore the persistent back pain occurred after the operation. PPSF, as a minimally invasive fixation technique, further enhances spinal stability and alleviates symptoms after infection control by PEDD, and provides a firm fixation of the spine through the minor trauma. Tsunemasa also suggested that PPSF should be considered as an additional surgery for patients with infectious spondylitis who underwent percutaneous drainage lavage in the presence of persistent postoperative low back pain or refractory pathogens that are not sensitive to antibiotics[18]. In our study, we demonstrate that PEDD can alleviate the pain and the additional PPSF can improve the outcome on this basis.

In addition, we believe that the application of PPSF can bring long-term benefits to patients. Infectious spinal inflammation inevitably leads to bone destruction, long term spontaneous intervertebral fusion and spinal deformity. Also, chronic spinal instability can cause underlying pain and neurological symptoms. Adequate isolation of infected segments is therefore essential for the management of infectious spinal 
inflammation [12]. Deininger et al. demonstrated that after posterior PPSF alone, the mean Cobb Angle increased by $3.4^{\circ}$ in during the 9 months follow-up period. Pee used anterior lesion removal and bone graft fusion with posterior PPSF to treat spondylodiscitis. The average follow-up time was 35.8 months. $91.3 \%$ of bone union and an average lordosis change of $4.5 \pm 9.0^{\circ}$ were reported. Nasto reported an average increase in Cobb Angle by $1.86 \pm 4.78^{\circ}$ during the 9 months follow-up period after simple posterior PPSF $[12,13,19,20]$. In the above studies, the intervertebral space was either treated by bone grafting or left untouched. However, in our study, the lesions in the intervertebral space were cleared without bone grafting. The local cavity is inevitably formed after the operation, which undoubtedly destroys the stability of the spine and promotes the development of spinal deformity. This may be the reason why kyphosis was developed in Ito's patients after PEDD treatment alone (the Cobb Angle increased by an average of $12^{\circ}$ ). However, the purpose of both conservative treatments using thoracolumbar braces and internal fixation devices is to fix infected segments, promote spontaneous healing, maintain spinal stability and slow down the progression of spinal deformity. Although the treatment of infectious spinal inflammation with posterior internal fixation remains controversial, studies have shown that posterior internal fixation provides better orthopedic results and promotes faster fusion of adjacent vertebrae, without increasing the risk of infection[13]. In our study spanning an average follow-up period of $7.84 \pm 4.29$ months, local Cobb Angle in patients increased by $3.9 \pm 6.7^{\circ}$, and the intervertebral bridge or fusion occurred in $90.9 \%$ of the patients. Therefore, we believe that the combination of PPSF and PEDD can be more effective in delaying the progress of kyphosis than PEDD alone, and providing better long-term efficacy to patients. However, a larger sample size and control group may be required to determine the effect of PEDD combined with PPSF on the long-term kyphosis deformity and stability.

There are many minimally invasive surgical methods alternative to open surgery in clinical practice. The main objective is to remove diseased tissue while minimizing trauma occurrence and performing subsequent microbial culture for the tissue. For infectious spinal inflammation, identification of pathogen species, while not necessary for diagnosis, is essential for successful treatment[21]. Blood culture and tissue culture are common methods to diagnose pathogenic bacteria. In general, the positive rate of blood culture can reach to $50 \%[6]$, while the positive rate of tissue culture can reach to $70 \%[8]$. In this group, 7 of the 33 patients tested positive for blood culture (21.2\%), while $24(72.7 \%)$ tested positive for intraoperative tissue culture through PEDD. Clinically, needle aspiration pathology is often used or pathology study is performed using CT, X-ray or ultrasound. However, the positive rates of tissue culture vary a lot according to methods used. Fouquet achieved a $36 \%$ positive rate through needle culture. Yang obtained a positive rate of $47 \%$ for CT guided biopsy. Staatz obtained a positive rate of $76 \%$ for CT guided biopsy. Chew and Kline achieved a 91\% positive rate [21-24] of CT guided biopsy in 43 patients with active infection. Yang pointed out that there were too many factors that might lead to a negative result for culturing, such as too few samples and sampling errors for the needle aspiration biopsy and imaging guided biopsy. In the literature of PEDD, the positive rate of pathological culture of PEDD can reach to $80-90 \%$ [21]. The advantages of PEDD include the direct extraction of pathological tissues, guarantee of sample size, reduction of sampling errors, and avoiding excessive radiographic guidance, 
huge trauma and potential complications caused by open surgery. Higher positive rate of culture can be obtained through PEDD, and early identification of pathogenic strains can also greatly reduce the likelihood of patients needing further open surgery. In our study, the positive rates of blood culture and tissue culture were low, which may be related to the fact that patients received antibiotics before surgery. Kim's study showed that the negative results of microbial culture were closely related to the use and length of antibiotics[25]. However, while reducing the positive rate of microbial culture, the application of inappropriate antibiotics can lead to the colonization of bacteria resistant to the whole class of antibiotics. More importantly, the colonization of antibiotic-resistant pathogens may increase the risk of subsequent infection with these highly toxic microorganisms. Current research suggests that patients with spinal infections who undergo frequent biopsies or surgery should not be treated with routine antibiotics[26]. Therefore, we believe that early identification of infectious strains is particularly critical. PEDD can achieve debridement and high positive rate for microbial culture.

In accordance with Yang's study, we used a large amount of dilute Beta iodized salt water with a concentration of $3.5 \%$ during the operation to obtain the optimal bactericidal effect[27]. In the previous literature on PEDD, the drainage tube was mostly implanted into the intervertebral disc area with the negative pressure after the operation, focus on the intervertebral space drainage to further clear the infected lesions[21, 27-30]. Compared with the previous study, we focus on the postoperative irrigation. In this study, a disposable epidural anesthesia catheter was inserted through the channel as an inlet pipe and an outlet pipe with a diameter of about $3.5 \mathrm{~mm}$. Walters infected the intervertebral space of the sheep with staphylococcus aureus and then applied cefazolin to it. Finally, the concentration of antibiotics in the blood was significantly higher than that in the annulus fibrosus, and there was no significant correlation between the two concentrations[31]. This suggests that when intra - disc infection occurs, antibiotics cannot reach the affected area no matter how high the intravenous antibiotic concentration is. This also reflects the limitations of conservative treatment for infectious spinal disease. However, we placed double tubes under the channels for postoperative continuous lavage and drainage, the inlet pipe was removed until the rinsing solution was clear and the outlet pipe was retained for drainage. For patients with positive microbial culture results after the operation, they were given relatively sensitive antibiotic irrigation through the inlet pipe. The antibiotic can reach the disc area directly, which can obviously improve the drug concentration in the lesion area. At the same time, local application of antibiotics, drugs do not go through the portal vein system, avoid long-term use of antibiotics side effects. After a large amount of intraoperative flushing to remove inflammation and diseased tissues, the continuous application of local drugs after the operation will undoubtedly enable infectious spinal inflammation to be further controlled.

\section{Limitations}

In this study, only patients with single segmental infection were studied, and patients with multisegmental infection were not included in the criterion. The efficacy of PEDD combined with PPSF for patients with multi-segmental infection still needs to be further analyzed. All patients in the study had received antibiotics before surgery, which may affect the positive rate of microbial culture. There was not 
enough sample for long-term follow-up to determine the degree of change and spontaneous fusion of patients with long-term kyphosis after surgery.

\section{Conclusion}

PEDD in combination with PPSF for the treatment of infectious spine inflammation can significantly reduce perioperative complications and surgical risk. Good curative effect for single segment infectious spondylitis was achieved, disregarding patient's condition with paravertebral abscess and epidural abscess. The combination has been shown to alleviate pain, reconstruct or maintain spinal stability, slow the progression of deformity, and promote spontaneous fusion of adjacent vertebral bodies. For patients with infectious spondylitis whose physical state is difficult to endure open surgery, the combination of PEDD and PPSF can achieve satisfactory results.

\section{Abbreviations}

PEDD: Percutaneous Endoscopic Debridement and Drainage

PPSF: Percutaneous Pedicle Screw Fixation

\section{Declarations}

\section{Ethics declarations}

\section{Ethics approval and consent to participate}

This study was approved by the institutional review board/Ethics Committee of The First Affiliated Hospital of Harbin Medical University and was conducted in compliance with the guidelines of the Helsinki Declaration of 1975.

\section{Consent for publication}

Consent for publication has been obtained from that All patients in this study.

\section{Availability of data and materials}

The datasets used and/or analysed during the current study are available from the corresponding author on reasonable request.

\section{Competing interests}

The authors declare that they have no competing interests.

\section{Funding}


Not applicable

\section{Authors' contributions}

$\mathrm{JL}$ was a major contributor in writing the manuscript;

GA and YG performed the patients' demographic data collection;

XS and RW analyzed and interpreted the patient data regarding VAS, Frankle classification囚etc;

DL and YF consult relevant literature;

YW designed the study and put forward relevant guidance.

All authors read and approved the final manuscript.

\section{Acknowledgements}

Not applicable

\section{References}

1. Kourbeti IS, Tsiodras S, Boumpas DT. Spinal infections: evolving concepts. Curr Opin Rheumatol. 2008;20:471-9.

2. Eismont FJ, Bohlman HH, Soni PL, Goldberg VM, Freehafer AA. Pyogenic and fungal vertebral osteomyelitis with paralysis. J Bone Joint Surg Am. 1983;65:19-29.

3. Carragee EJ. Pyogenic vertebral osteomyelitis. J Bone Joint Surg Am. 1997;79:874-80.

4. McHenry MC, Easley KA, Locker GA. Vertebral osteomyelitis: long-term outcome for 253 patients from 7 Cleveland-area hospitals. Clin Infect Dis. 2002;34:1342-50.

5. Gupta A, Kowalski TJ, Osmon DR, Enzler M, Steckelberg JM, Huddleston PM, et al. Long-term outcome of pyogenic vertebral osteomyelitis: a cohort study of 260 patients. Open Forum Infect Dis. 2014; 1:ofu107.

6. Govender S. Spinal infections. J Bone Joint Surg Br. 2005;87:1454-8.

7. Mylona E, Samarkos M, Kakalou E, Fanourgiakis P, Skoutelis A. Pyogenic vertebral osteomyelitis: a systematic review of clinical characteristics. Semin Arthritis Rheum. 2009;39:10-7.

8. Skaf GS, Domloj NT, Fehlings MG, Bouclaous CH, Sabbagh AS, Kanafani ZA, et al. Pyogenic spondylodiscitis: an overview. J Infect Public Health. 2010;3:5-16.

9. Hanaoka N, Kawasaki Y, Sakai T, Nakamura T, Nanamori K, Nakamura E, et al. Percutaneous drainage and continuous irrigation in patients with severe pyogenic spondylitis, abscess formation, and marked bone destruction. J Neurosurg Spine. 2006;4:374-9.

10. Madhavan K, Vanni S, Williams SK. Direct lateral retroperitoneal approach for the surgical treatment of lumbar discitis and osteomyelitis. Neurosurg Focus. 2014;37:art. no. E5-5art. no. E5.. 
11. Sharan AD, Przybylski GJ. Single-stage autogenous bone grafting and internal fixation in the surgical management of pyogenic discitis and vertebral osteomyelitis. Journal of neurosurgery. 2001;94:1-7.

12. Nasto LA, Colangelo D, Mazzotta V, Di Meco E, Neri V, Nasto RA, et al. Is posterior percutaneous screw-rod instrumentation a safe and effective alternative approach to TLSO rigid bracing for singlelevel pyogenic spondylodiscitis? Results of a retrospective cohort analysis. Spine J. 2014;14:113946.

13. Pee YH, Park JD, Choi YG, Lee SH. Anterior debridement and fusion followed by posterior pedicle screw fixation in pyogenic spondylodiscitis: autologous iliac bone strut versus cage. J Neurosurg Spine. 2008;8:405-12.

14. Ruf M, Stoltze D, Merk HarryR. Michael.A. Treatment of Vertebral Osteomyelitis by Radical Debridement and Stabilization Using Titanium Mesh Cages. Spine (Phila Pa 1976). 2007;32:E2755 E280.

15. Glassman SD, Hartman MJ, Campbell MJ, Johnson JR, Dimar JR, Carreon LY. Treatment of pyogenic vertebral osteomyelitis with anterior debridement and fusion followed by delayed posterior spinal fusion. Spine (Phila Pa 1976). 2004;29:326 - 32; discussion 332-0..

16. janat00218-0176.

17. Wang YC, Wong CB, Wang IC, Fu TS, Chen LH, Chen WJ. Exposure of Prebiopsy Antibiotics Influence Bacteriological Diagnosis and Clinical Outcomes in Patients With Infectious Spondylitis. Medicine. 2016;95:e3343.

18. Yamada K, Sato K, Gotoh M, Matsubara T, Nagata K, Shiba N. Clinical outcomes of percutaneous suction aspiration and drainage for the treatment of infective spondylodiscitis with paravertebral or epidural abscess. The spine journal: official journal of the North American Spine Society. 2018;18:1558-69.

19. Vougioukas VI, Hubbe U, Unfried MI, Deininger MH. Minimally invasive dorsal percutaneous spondylodesis for the treatment of adult pyogenic spondylodiscitis. Acta Neurochir (Wien). 2009;151:1451-7.

20. Lin TY, Tsai TT, Lu ML, Niu CC, Hsieh MK, Fu TS, et al. Comparison of two-stage open versus percutaneous pedicle screw fixation in treating pyogenic spondylodiscitis. BMC Musculoskelet Disord. 2014;15:443.

21. Yang SC, Fu TS, Chen LH, Chen WJ, Tu YK. Identifying pathogens of spondylodiscitis: percutaneous endoscopy or CT-guided biopsy. Clin Orthop Relat Res. 2008;466:3086-92.

22. Fouquet $B$, Goupille $P$, Jattiot $F$, Cotty $P$, Lapierre F, Valat JP, et al. Discitis after lumbar disc surgery. Features of "aseptic" and "septic" forms. Spine (Phila Pa 1976). 1992;17:356-8.

23. Staatz G, Adam GB, Keulers P, Vorwerk D, Günther RW. Spondylodiskitic abscesses: CT-guided percutaneous catheter drainage. Radiology. 1998;208:363-7.

24. Chew FS, Kline MJ. Diagnostic yield of CT-guided percutaneous aspiration procedures in suspected spontaneous infectious diskitis. Radiology. 2001;218:211-4. 
25. Kim CJ, Song KH, Park WB, Kim ES, Park SW, Kim HB, et al. Microbiologically and clinically diagnosed vertebral osteomyelitis: impact of prior antibiotic exposure. Antimicrob Agents Chemother. 2012;56:2122-4.

26. Kollef $M H$, Sherman G, Ward S, Fraser VJ. Inadequate antimicrobial treatment of infections: a risk factor for hospital mortality among critically ill patients. Chest. 1999;115:462-74.

27. Yang SC, Chen WJ, Chen HS, Kao YH, Yu SW, Tu YK. Extended indications of percutaneous endoscopic lavage and drainage for the treatment of lumbar infectious spondylitis. Eur Spine J. 2014;23:846-53.

28. Ito M, Abumi K, Kotani Y, Kadoya K, Minami A. Clinical outcome of posterolateral endoscopic surgery for pyogenic spondylodiscitis: results of 15 patients with serious comorbid conditions. Spine (Phila Pa 1976). 2007;32:200-6.

29. Wang X, Zhou S, Bian Z, Li M, Jiang W, Hou C, et al. Unilateral percutaneous endoscopic debridement and drainage for lumbar infectious spondylitis. J Orthop Surg Res. 2018;13:306.

30. Yang SC, Fu TS, Chen LH, Niu CC, Lai PL, Chen WJ. Percutaneous endoscopic discectomy and drainage for infectious spondylitis. Int Orthop. 2007;31:367-73.

31. Walters R, Rahmat R, Fraser R, Moore R. Preventing and treating discitis: cephazolin penetration in ovine lumbar intervertebral disc. Eur Spine J. 2006;15:1397-403.

\section{Tables}

Table1: Patient demographic data 


\begin{tabular}{|c|c|c|c|c|c|c|c|c|c|}
\hline \multicolumn{4}{|c|}{ nder age segment ASA } & \multirow{2}{*}{$\begin{array}{c}\begin{array}{c}\text { Charlson complication } \\
\text { index }\end{array} \\
2\end{array}$} & \multirow{2}{*}{$\frac{\begin{array}{c}\text { paravertebral } \\
\text { abscess }\end{array}}{\mathrm{N}}$} & \multirow{2}{*}{$\frac{\begin{array}{c}\text { Epidural } \\
\text { abscess }\end{array}}{\mathrm{N}}$} & \multirow{2}{*}{$\begin{array}{c}\text { Preoperative } \\
\text { VAS } \\
8\end{array}$} & \multirow{2}{*}{$\begin{array}{c}\text { Preoperative } \\
\mathrm{ESR}(\mathrm{mm} / \mathrm{h})\end{array}$} & \multirow{2}{*}{$\begin{array}{c}\begin{array}{c}\text { Preoperative } \\
\mathrm{CRP}(\mathrm{mg} / \mathrm{L})\end{array} \\
117\end{array}$} \\
\hline M & 77 & $\mathrm{~L} 3 / 4$ & 3 & & & & & & \\
\hline $\mathrm{F}$ & 70 & $\mathrm{~L} 2 / 3$ & 3 & 0 & $\mathrm{~N}$ & $\mathrm{~N}$ & 7 & 58 & 95.1 \\
\hline $\mathrm{M}$ & 80 & $\mathrm{~L} 2 / 3$ & 3 & 2 & $\mathrm{~N}$ & $\mathrm{~N}$ & 7 & 61 & 94.2 \\
\hline $\mathrm{F}$ & 75 & $\mathrm{~L} 4 / 5$ & 3 & 5 & $\mathrm{Y}$ & $\mathrm{N}$ & 8 & 77 & 93.1 \\
\hline $\mathrm{F}$ & 69 & $\mathrm{~L} 2 / 3$ & 3 & 1 & $\mathrm{~N}$ & $\mathrm{~N}$ & 6 & 59 & 84.5 \\
\hline $\mathrm{M}$ & 46 & $\mathrm{~L} 3 / 4$ & 2 & 0 & $\mathrm{~N}$ & $\mathrm{~N}$ & 7 & 72 & 83.1 \\
\hline $\mathrm{M}$ & 84 & $\mathrm{~L} 3 / 4$ & 3 & 1 & $\mathrm{Y}$ & $\mathrm{Y}$ & 9 & 79 & 96.7 \\
\hline $\mathrm{M}$ & 72 & $\mathrm{~L} 3 / 4$ & 3 & 0 & $\mathrm{~N}$ & $\mathrm{~N}$ & 6 & 64 & 111 \\
\hline $\mathrm{M}$ & 85 & $\mathrm{~L} 3 / 4$ & 3 & 3 & $\mathrm{~N}$ & $\mathrm{Y}$ & 7 & 57 & 85.3 \\
\hline $\mathrm{M}$ & 75 & $\mathrm{~L} 4 / 5$ & 3 & 1 & $\mathrm{~N}$ & $\mathrm{~N}$ & 7 & 85 & 87.2 \\
\hline $\mathrm{M}$ & 69 & $\mathrm{~L} 4 / 5$ & 2 & 1 & $\mathrm{~N}$ & $\mathrm{~N}$ & 7 & 62 & 91 \\
\hline $\mathrm{F}$ & 81 & $\mathrm{~L} 3 / 4$ & 3 & 2 & $\mathrm{~N}$ & $\mathrm{Y}$ & 7 & 65 & 105 \\
\hline $\mathrm{M}$ & 77 & L5 / S1 & 3 & 3 & $\mathrm{~N}$ & $\mathrm{~N}$ & 6 & 70 & 86.2 \\
\hline $\mathrm{M}$ & 58 & $\mathrm{~L} 4 / 5$ & 2 & 4 & $\mathrm{Y}$ & $\mathrm{N}$ & 8 & 66 & 88 \\
\hline $\mathrm{F}$ & 60 & $\mathrm{~L} 3 / 4$ & 2 & 0 & $\mathrm{Y}$ & $\mathrm{N}$ & 7 & 90 & 103 \\
\hline $\mathrm{F}$ & 86 & $\mathrm{~L} 4 / 5$ & 3 & 0 & $\mathrm{~N}$ & $\mathrm{Y}$ & 7 & 55 & 75 \\
\hline $\mathrm{M}$ & 78 & $\mathrm{~L} 2 / 3$ & 2 & 4 & $\mathrm{~N}$ & $\mathrm{~N}$ & 6 & 62 & 87.2 \\
\hline $\mathrm{F}$ & 80 & $\mathrm{~L} 2 / 3$ & 3 & 1 & $\mathrm{Y}$ & $\mathrm{N}$ & 8 & 70 & 94 \\
\hline $\mathrm{M}$ & 75 & $\mathrm{~L} 3 / 4$ & 3 & 2 & $\mathrm{Y}$ & $\mathrm{N}$ & 8 & 61 & 87 \\
\hline $\mathrm{M}$ & 67 & $\mathrm{~L} 2 / 3$ & 3 & 3 & $\mathrm{Y}$ & $\mathrm{Y}$ & 9 & 74 & 90 \\
\hline $\mathrm{F}$ & 29 & $\mathrm{~L} 4 / 5$ & 1 & 0 & $\mathrm{~N}$ & $\mathrm{~N}$ & 7 & 69 & 92.3 \\
\hline $\mathrm{M}$ & 75 & $\mathrm{~L} 2 / 3$ & 2 & 2 & $\mathrm{~N}$ & $\mathrm{Y}$ & 8 & 59 & 85.6 \\
\hline $\mathrm{F}$ & 89 & $\mathrm{~L} 3 / 4$ & 2 & 3 & $\mathrm{~N}$ & $\mathrm{~N}$ & 7 & 62 & 78.3 \\
\hline $\mathrm{M}$ & 79 & $\mathrm{~L} 4 / 5$ & 2 & 0 & $\mathrm{~N}$ & $\mathrm{~N}$ & 6 & 58 & 80 \\
\hline $\mathrm{M}$ & 33 & $\mathrm{~L} 2 / 3$ & 1 & 1 & $\mathrm{~N}$ & $\mathrm{~N}$ & 6 & 71 & 93 \\
\hline $\mathrm{F}$ & 87 & $\mathrm{~L} 3 / 4$ & 2 & 0 & $\mathrm{Y}$ & $\mathrm{N}$ & 7 & 64 & 79 \\
\hline $\mathrm{M}$ & 65 & $\mathrm{~L} 4 / 5$ & 3 & 3 & $\mathrm{~N}$ & $\mathrm{Y}$ & 7 & 73 & 93 \\
\hline $\mathrm{M}$ & 73 & L5 / S1 & 3 & 1 & $\mathrm{~N}$ & $\mathrm{~N}$ & 7 & 62 & 84.5 \\
\hline $\mathrm{M}$ & 77 & $\mathrm{~L} 3 / 4$ & 3 & 1 & $\mathrm{Y}$ & $\mathrm{N}$ & 8 & 86 & 104 \\
\hline $\mathrm{F}$ & 73 & $\mathrm{~L} 4 / 5$ & 3 & 4 & $\mathrm{~N}$ & $\mathrm{~N}$ & 6 & 73 & 95.3 \\
\hline $\mathrm{M}$ & 65 & L5 / S1 & 2 & 0 & $\mathrm{~N}$ & $\mathrm{Y}$ & 8 & 68 & 96.1 \\
\hline $\mathrm{F}$ & 53 & $\mathrm{~L} 3 / 4$ & 2 & 3 & $\mathrm{Y}$ & $\mathrm{N}$ & 8 & 72 & 84.5 \\
\hline
\end{tabular}


Table2: ODOM's criteria

\begin{tabular}{lcccc}
\hline & Excellent & Good & Fair & Poor \\
\hline Odom 's standard & 22 & 11 & 0 & 0 \\
& & & & \\
\hline
\end{tabular}

Table3: neurological function evaluation

\begin{tabular}{|c|c|c|c|c|c|c|}
\hline \multicolumn{7}{|c|}{ Frankel classification } \\
\hline \multicolumn{2}{|c|}{ Modified Frankel score } & \multicolumn{5}{|c|}{ Postoperative } \\
\hline & & $\mathrm{C} 2$ & D3 & D4 & D5 & $\mathrm{E}$ \\
\hline \multirow[t]{5}{*}{ preoperative } & $\mathrm{C} 2$ & 1 & 2 & 2 & 1 & \\
\hline & D3 & & & 1 & 1 & \\
\hline & D4 & & & & 3 & \\
\hline & D5 & & & & 1 & \\
\hline & E & & & & & 21 \\
\hline
\end{tabular}

Table 4: biological analysis of causative organisms

$\frac{\text { Pathological tissue culture results }}{\text { Culture positive patient }}$

\begin{tabular}{cc}
\hline Only the blood culture was positive & $3(9 \%)$ \\
\hline Only tissue culture was positive & $21(63.6 \%)$. \\
\hline All positive & $4(12.1 \%)$ \\
\hline All negative & $6(18.1 \%)$ \\
\hline
\end{tabular}

Table 5: Causative organism Strains 
Pathogenic strains

\begin{tabular}{ll}
\hline MRSA & 9 \\
\hline Staphylococcus aureus & 6 \\
\hline Streptococcus pneumoniae & 4 \\
\hline Streptococcus green & 3 \\
\hline Mycobacterium tuberculosis & 3 \\
\hline brucella & 2 \\
\hline
\end{tabular}

\section{Figures}

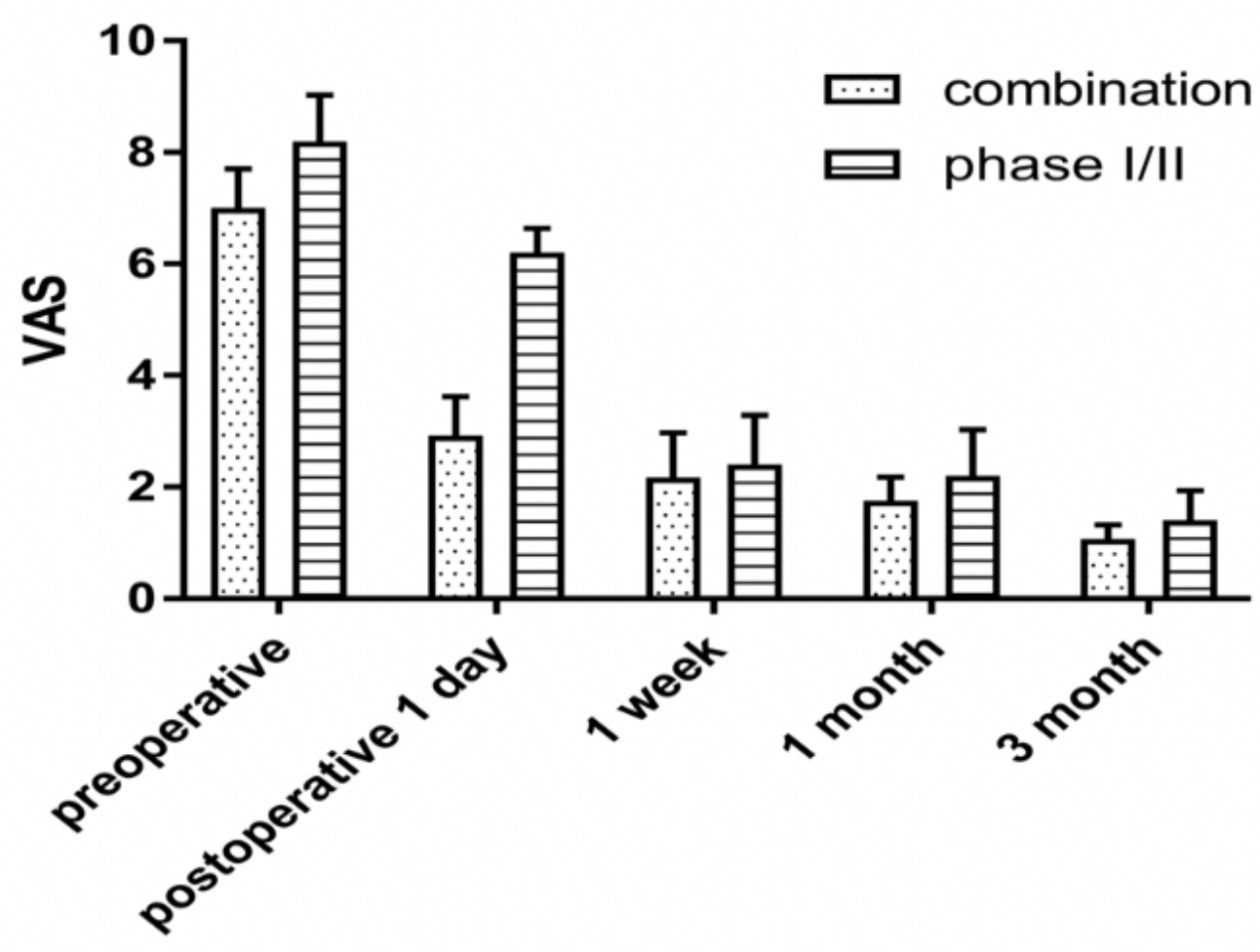

\begin{tabular}{lccccc}
\hline VAS & preoperate & Postoperative 1 day & 1 week & 1 month & 3 month \\
\hline Combination & 7 & 2.92 & 2.17 & 1.75 & 1.07 \\
Phase I/II & 8.2 & 6.2 & 2.4 & 2.2 & 1.4 \\
\hline
\end{tabular}

Figure 1

VAS 


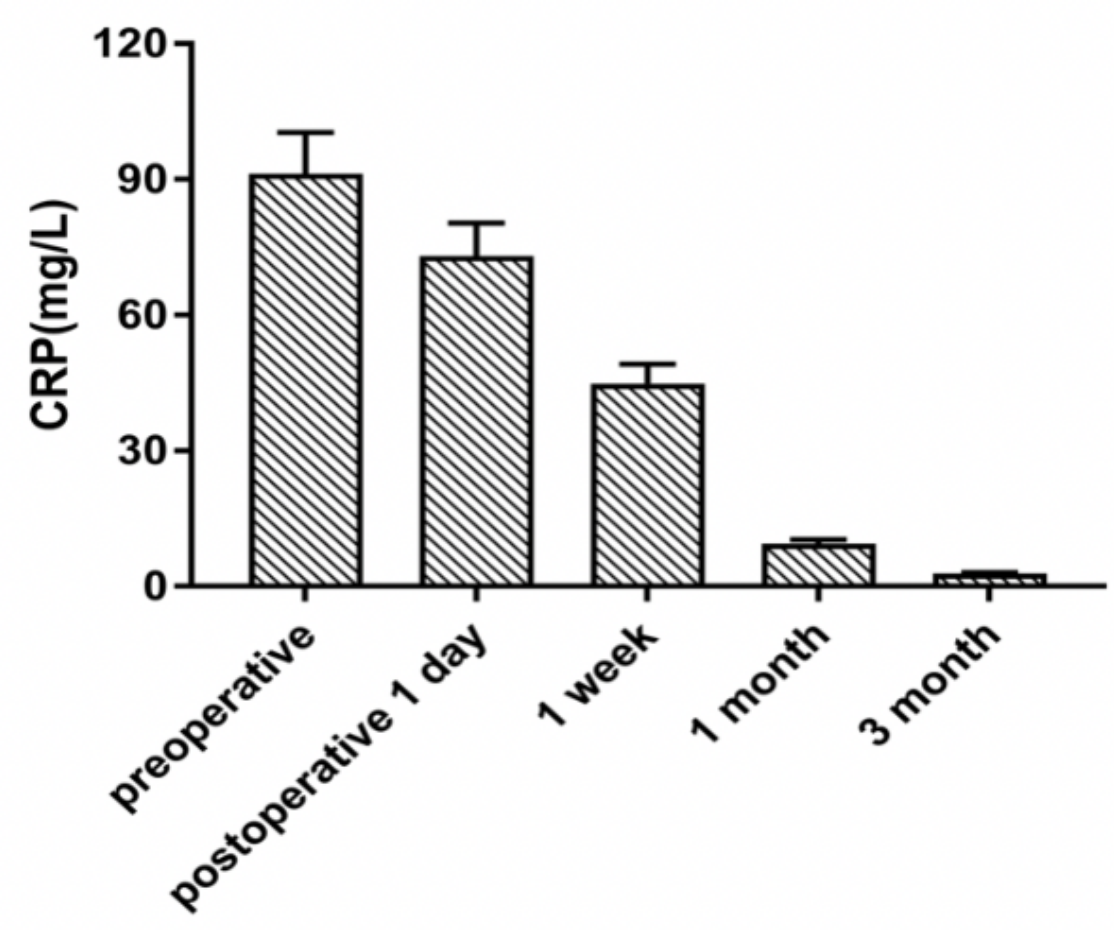

\begin{tabular}{llllll}
\hline & preoperative & Postoperative 1 day & 1 week & 1 month & 3 month \\
\hline $\mathrm{CRP}(\mathrm{mg} / \mathrm{L})$ & $91.3 \pm 9.09$ & $73.04 \pm 7.27$ & $44.82 \pm 4.36$ & $9.46 \pm 0.87$ & $2.83 \pm 0.26$ \\
\hline
\end{tabular}

Figure 2

CRP 


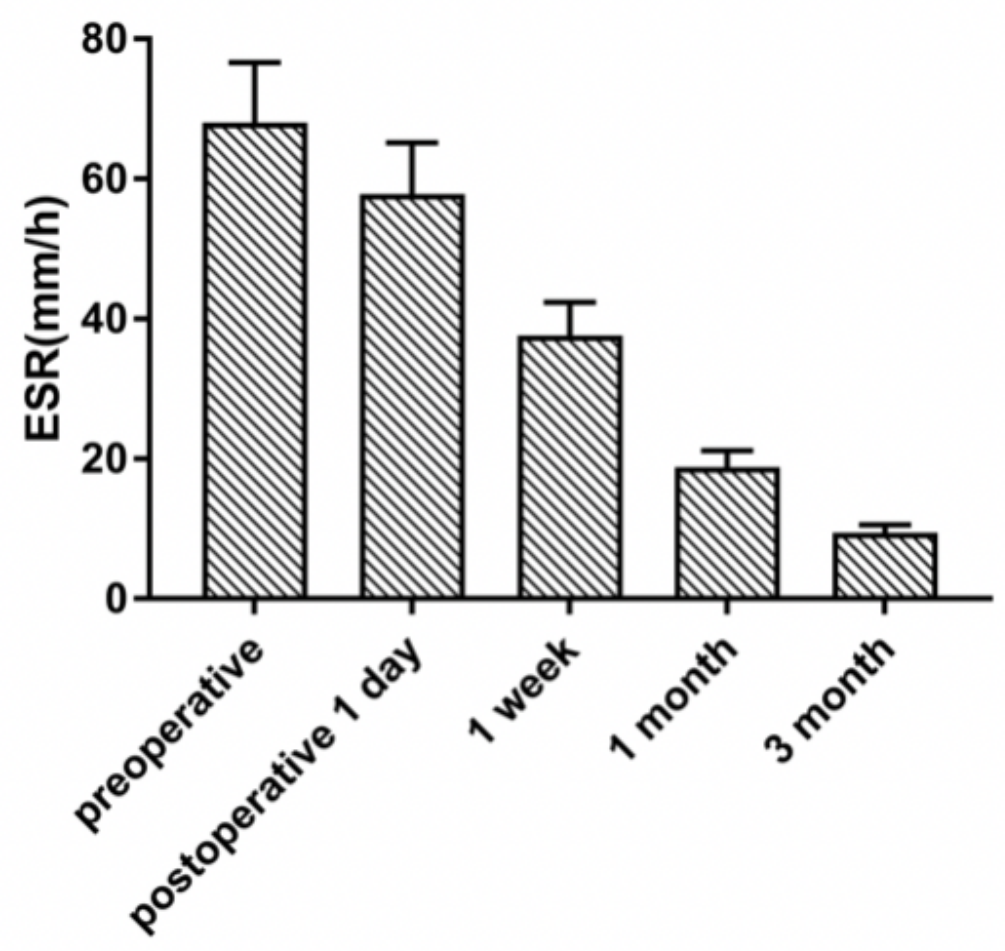

\begin{tabular}{|c|c|c|c|c|c|}
\hline & preoperative & $\begin{array}{l}\text { Postoperative } \\
\text { day }\end{array}$ & 1 week & 1 month & 3 month \\
\hline $\mathrm{ESR}(\mathrm{mm} / \mathrm{h})$ & $68.06 \pm 8.60$ & $57.85 \pm 7.31$ & $37.60 \pm 4.75$ & $18.80 \pm 2.37$ & $9.40 \pm 1.18$ \\
\hline
\end{tabular}

Figure 3

ESR 

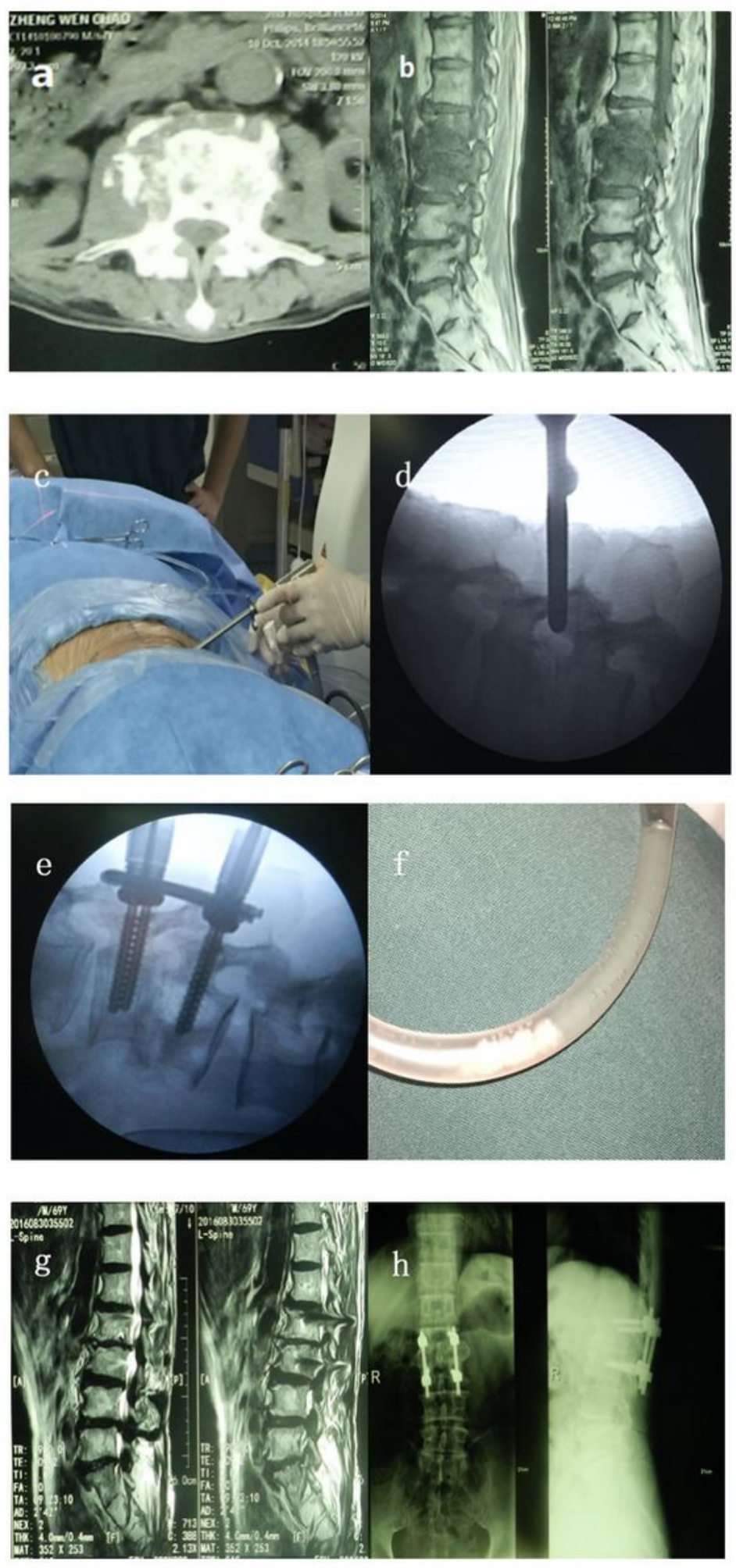

\section{Figure 4}

Male, 67 years old, low back pain for 9 years, aggravating for 3 months. Years of history of renal failure, poor physical condition, ASA grade III, Charlson complication index score 3. CT showed L2-3 vertebra destruction (a), and MRI showed signal of L2-3 vertebral and intervertebral space changed, and fuzzy shadows were observed in spinal canal (b). Patients underwent PEDD and PPSF surgery (c囚d囚e), and there was intermittent flow of flocculent in the outlet pipe (f). After the operation, the patient's pain was 
significantly relieved, and MRI (g) was performed. No recurrence or surgical complications were found during the follow-up for two years. X-ray examination showed spontaneous fusion in L2-3, and local Cobb Angle increased by 4.1 degrees (h). 